\title{
Exportin T and Exportin 5: tRNA and miRNA biogenesis - and beyond
}

\author{
Matthias S. Leisegang ${ }^{1, a}$, Roman Martin ${ }^{1,2, a}$, \\ Ana S. Ramírez ${ }^{1, a}$ and Markus T. Bohnsack ${ }^{1-3, *}$ \\ ${ }^{1}$ Institute for Molecular Biosciences, Goethe University, \\ D-60438 Frankfurt, Germany \\ ${ }^{2}$ Centre for Biochemistry and Molecular Cell Biology, \\ Göttingen University, D-37073 Göttingen, Germany \\ ${ }^{3}$ Cluster of Excellence Frankfurt, Goethe University, \\ D-60438 Frankfurt, Germany \\ * Corresponding author \\ e-mail: Markus.Bohnsack@med.uni-goettingen.de
}

\begin{abstract}
The biogenesis of most eukaryotic kinds of RNA requires nuclear export, which is mediated by a variety of specific nuclear transport receptors. The nuclear export receptors Exportin-t (Exp-t) and Exportin 5 (Exp5), and their homologues, are involved in the export of transfer RNA to the cytoplasm. Exp5 is further involved in additional nucleocytoplasmic transport pathways, which include nuclear export of microRNA precursors (pre-miRNAs) and pre-60S ribosomal subunits. Inactivation of Exp5 results in nuclear accumulation of pre-miRNAs and perturbation of gene expression, and its mutation was recently found in malignant diseases. Here, we compare the cellular function of Exp5 and Exp-t with focus on Exp5 substrates and its role in diseases.
\end{abstract}

Keywords: gene expression; nuclear export; ribosome; RNA transport; RNP biogenesis; translation.

The defining feature of the eukaryotic cell is its nucleus, which is separated from the cytoplasm by the nuclear envelope (NE). Nuclear pore complexes (NPCs) are embedded in the NE and allow the exchange of material between the two compartments (see Güttler and Görlich, 2011, and references therein). NPCs are composed of multiple copies of $\sim 30$ nucleoporin proteins, many of which contain phenylalanine-glycine repeats that constitute the NPC's permeability barrier. This barrier permits the free passage of small molecules, but restricts that of material greater than $20-40 \mathrm{kDa}$ in molecular weight. Most macromolecules and complexes therefore require nuclear transport receptors (NTRs) to pass the NPC. NTRs interact with both their cargoes and NPCs to allow passage of even very large

aThese authors contributed equally to this article. cargoes. The majority of NTRs belong to the Importin $\beta$-family, which comprises import mediators (importins) as well as export mediators (exportins) (Güttler and Görlich, 2011).

Both exportins and importins shuttle between the nucleus and the cytoplasm and recruit their cargoes on one side of the NE to unload it in the other compartment. Directionality of transport is brought about by a gradient of the small, Ras-like GTPase Ran (Gsp1 in yeast) in its GTPbound form (RanGTP). The high concentration of RanGTP in the nucleus is due to its nucleotide (nt) exchange factor, which localises to chromatin, while the GTPase activating protein RanGAP and its co-factors are cytoplasmic. Exportins cooperatively bind the export signal of their cargoes in the nucleus together with RanGTP and translocate to the cytoplasm as RanGTP exportin cargo complexes. After export, hydrolysis of the Ran-bound GTP leads to disassembly of the export complex. In contrast, importins bind their cargoes in the cytoplasm and release them upon binding of nuclear RanGTP.

Following their synthesis in the nucleus, many kinds of RNA require export (mostly as RNPs or precursors) to the cytoplasm, including mRNAs, rRNAs and small RNAs, such as snRNAs, miRNAs as well as tRNAs (Köhler and Hurt, 2007). mRNA export is not regulated by the Ran system, but instead mediated by the conserved export receptor Mex67-Mtr2 heterodimer in yeast (NXF1-NXT1 or TAP-p15, in metazoans), which in turn is recruited by other adaptor proteins such as Yral (termed Aly/REF in metazoans). These export complexes are disassembled by the cytoplasmic RNA helicase Dbp5 (Ddx19). In ribosome biogenesis, (pre-) rRNA is exported as part of pre-ribosomal subunits (reviewed by Köhler and Hurt, 2007). Export of pre-60S particles is best understood and requires the Ran system as well as Ran-independent NPC-interacting export factors, such as Arx1, Ecm1 and the mRNA export factors Mex67-Mtr2. The exportin chromosome region maintenance 1 (CRM1) is recruited via the adaptor protein $\mathrm{Nmd} 3$ and is released upon hydrolysis of Ran-bound GTP; Nmd3 is removed from the particle by the GTPase protein Lsg1. Both the mode of recruitment and release of the Mex67-Mtr2 heterodimer in pre-60S export are unclear so far. Export of snRNAs is mediated by CRM1 in complex with the adaptor protein PHAX (in a phosphorylated form) and the cap binding complex CBC. After translocation, the hydrolysis of Ran-bound GTP and dephosphorylation of PHAX induce complex disassembly and snRNA release (see Köhler and Hurt, 2007). 
Here, we focus on the two Importin $\beta$-like nuclear export receptors Exportin-t (Exp-t; homologues: XpoT, Los1, PAUSED) and Exportin 5 (Exp5; homologues: Xpo5, Msn5, HASTY), which can directly interact with RNA cargoes. Both receptors have been implicated in tRNA export, while Exp5 is required for multiple RNA and RNP export pathways. Structures of free Schizosaccharomyces pombe Exp-t or Exp-t in complex with tRNA and RanGTP have been solved (Cook et al., 2009), while for Exp5, only a complex with RanGTP and a pre-miRNA (Okada et al., 2009) is available. Both NTRs show the typical superhelical solenoid structure seen in all characterised members of the Importin $\beta$-family (see Güttler and Görlich, 2011). These NTRs consist of HEAT repeats, which comprise two $\alpha$-helices (denoted A and B) that are connected by a short loop. While the A-helix of each repeat contributes to the receptor's outer surface, which, in general, interacts with nucleoporins during NPC passage, the B-helices establish the concave inner surface that recruits RanGTP and, in most cases, provides binding sites for cargoes (Güttler and Görlich, 2011).

The tRNA-specific Exp-t contacts features that are structurally conserved among tRNAs (Cook et al., 2009). Generally, the common tertiary structure for all tRNAs is required for recognition by biogenesis factors and ribosomes in translation, while each class of tRNA harbours a characteristic and complex pattern of modifications as signature for specific aminoacyl tRNA synthetase recognition. Exp-t measures tRNA folding like a 'molecular ruler' (Cook et al., 2009; Güttler and Görlich, 2011). The regions recognised by Exp-t include

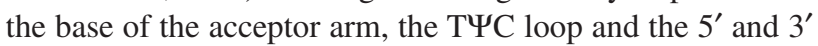
termini (including the $3^{\prime} \mathrm{CCA}$ ), which ensures that tRNA is correctly folded and processed before export occurs (Cook et al., 2009; Hopper et al., 2010). How exactly Exp-t/Los1 recognises tRNA modifications remains unclear. Regions of the molecule that vary between tRNAs, such as the hypervariable and anticodon loops, are not contacted by the receptor, in the latter case also allowing export of unspliced tRNAs during their biogenesis. While free Exp-t has an elongated form, RanGTP binding to Exp-t induces structural rearrangements resulting in a more closed structure of the receptor (Cook et al., 2009). Together with the direct RanGTP-tRNA interaction, the structural rearrangements contribute to cooperative binding of RanGTP and tRNA observed during export complex formation.

In contrast to Exp-t, Exp5 has various export substrates but, so far, only a structure of an export complex containing Exp5, RanGTP and a pre-miRNA is available (see below). In this structure, a positively charged surface of Ran contributes to cargo binding (Okada et al., 2009; Wang et al., 2011), similar as in the Exp-t export complex (Cook et al., 2009). This again explains the cooperativity observed for Exp5 upon export complex formation. As no structural data are available of an Exp5 complex containing a tRNA molecule, it remains unclear which molecular contacts are formed with the receptor. However, in vitro analyses already indicate that the mode of interaction is different from that of Exp-t. Interestingly, Exp5 can recruit aminoacylated tRNAs bound to the eukaryotic translation elongation factor $1 \mathrm{~A}(\mathrm{eEF} 1 \mathrm{~A})$ (Bohnsack et al., 2002; Calado et al., 2002). This indicates that the $3^{\prime}$ end of the tRNA is not protected in the Exp5 complex, but that even a large protein as eEF1A can be bound when the tRNA is aminoacylated. This seems in contrast to Exp-t, which directly interacts with and thereby probes for the presence of the CCA tail of the bound tRNA (Cook et al., 2009) and is thought not to allow an eEF1A interaction.

The different ways of RNA interaction of the two nuclear export receptors also influence the range of cargoes available for Exp5 and Exp-t. In case of tRNA biogenesis, the pathway as well as the roles of Exp-t and Exp5 and their orthologues Los1 and Msn5, respectively, are best understood in the yeast Saccharomyces cerevisiae (see Figure 1A; Hopper et al., 2010). Here, transcription is followed by $5^{\prime}$-leader sequence removal by RNase $\mathrm{P}$, which in a $\mathrm{La}$ (Lhp1) protein-dependent manner precedes 3' maturation mainly by RNase Z. More than 100 different nt modifications are known in tRNAs and many of these are introduced before primary export of the pre-tRNA by Los1. In yeast, splicing of intron-containing tRNAs occurs after nuclear export at the cytoplasmic face of outer mitochondrial membrane, while the process is thought to take place in the nucleus in vertebrates. Intron removal and modifications result in mature tRNAs that can be recognised and aminoacylated by the corresponding aminoacyl tRNA synthetases (Hopper et al., 2010). Interestingly, many tRNAs are re-imported into the nucleus by the importin Mtr10, where further modifications can be introduced and are controlled by aminoacyl tRNA synthetases during aminoacylation, before secondary export (Figure 1A). This nuclear state is thought to represent another level of quality control to ensure that tRNAs are properly folded and can specifically be recognised by the transport and ultimately the aminoacylation machinery. Los1 has been implicated in both export events, while Msn5 is thought to only be involved in secondary export (Hopper et al., 2010). However, as Msn5 and Los1 are not essential and double knockouts are viable, it is assumed that an additional mechanism for tRNA export could exist. One additional factor that plays a role in cytoplasmic tRNA localisation is the translation factor Tef1/2 (eEF1A). It remains to be shown whether Tef $1 / 2$ acts in export or rather sequestering of aminoacylated tRNA in the cytoplasm. Furthermore, the cytoplasmic protein Cex1 is thought to increase the efficiency of export complex disassembly by recruiting the GTPase activating protein Rna1/RanGAP.

Multiple levels of quality control have been found in tRNA biogenesis pathways. General mechanisms of RNA surveillance also control tRNA integrity. For example, the TRAMP complex and the exosome degrade misfolded or hypomodified tRNA precursors. In addition, the Lsm2-8 complex is possibly involved in quality control and the subsequent degradation by the exosome.

Important functions in the specific quality control of tRNAs are accomplished by the nuclear export receptors and nuclear as well as cytoplasmic aminoacyl tRNA synthetases (Hopper et al., 2010). Quality control steps include primary export, when Los1 probes for correct folding and processing of tRNAs, CCA addition and some modifications. Los1 allows introns in these tRNAs, while Exp5/Msn5 has been suggested 

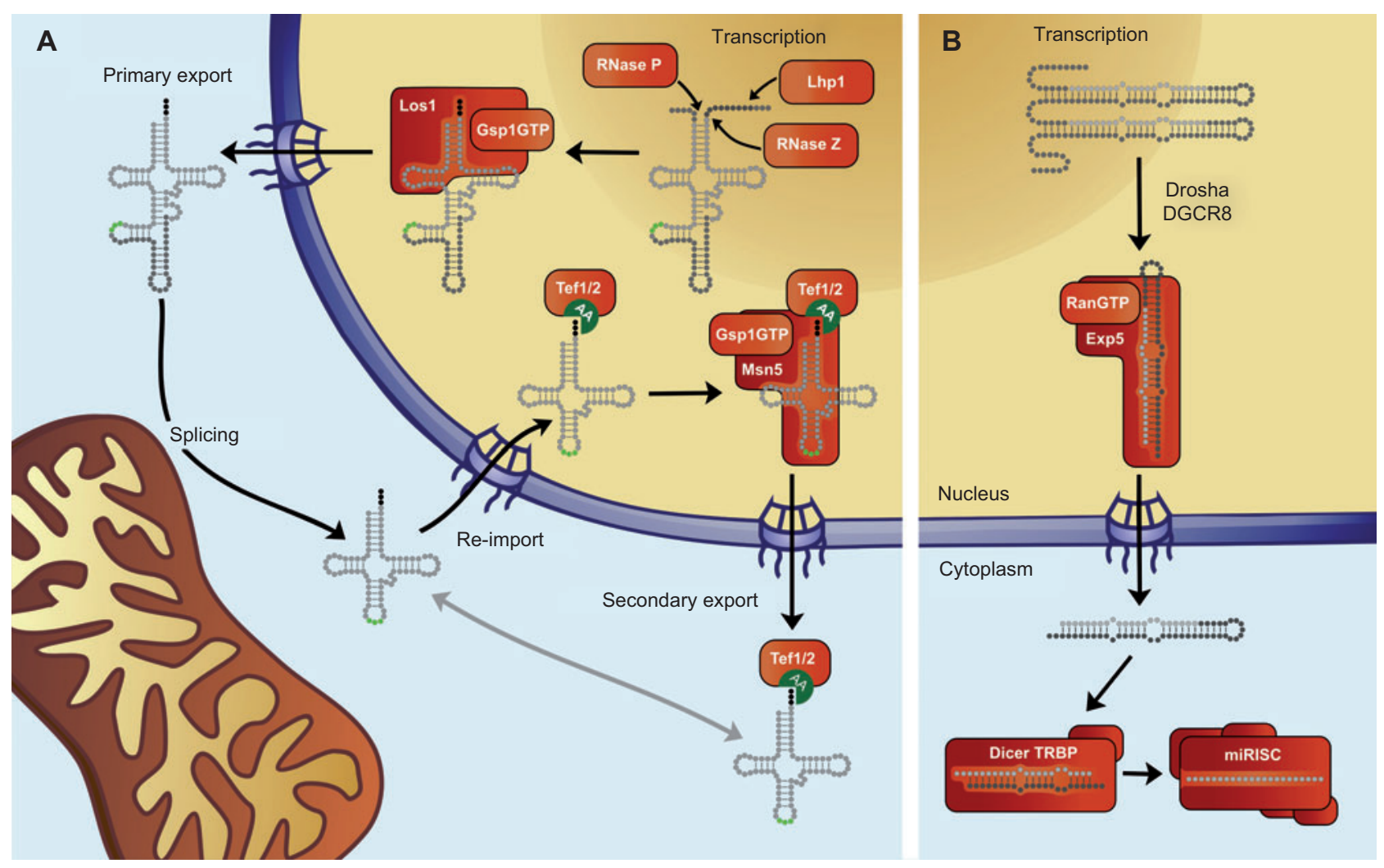

Figure 1 Biogenesis of tRNAs and miRNAs in eukaryotic cells.

Schematic representations of tRNA processing and maturation in yeast (A) and of the canonical miRNA biogenesis pathway in human cells (B) are shown. Mature RNAs are shown in grey; other nt of precursors in dark grey. In (A), the tRNA anticodon is indicated in light green, the 3'-terminal CCA in black and aminoacylation by 'AA' (dark green). Pathways are explained in the main text.

not to recognise unspliced tRNAs. In addition, quality control by aminoacylation is also thought to involve Tef1/2. The selective transport of biogenesis intermediates and mature tRNAs coupled to the dual localisation and high specificity of aminoacyl tRNA synthetases ensures the functionality of tRNAs and therefore translation fidelity.

Besides the export of tRNAs, a major function of Exp5 and many of its orthologues is nuclear export of microRNA precursors (pre-miRNAs). miRNAs are single-stranded, $\sim 22 \mathrm{nt}$ long RNAs derived from hairpin-containing precursor transcripts (see Miyoshi et al., 2010, and references therein). They serve as post-transcriptional regulators of protein-coding mRNAs, leading to translational repression or mRNA degradation. Up to 1000 different human miRNAs have been identified so far.

miRNAs are transcribed individually or as part of clusters, typically by RNA polymerase II. The primary miRNA transcripts contain the stem-loop structures, which are excised as pre-miRNAs by the RNase III Drosha and its co-factor DiGeorge syndrome critical region 8 (DGCR8) that form the core of the Microprocessor complex in the nucleus (Figure 1B). The dsRNA binding protein DGCR8 recognises single-stranded RNA sections at the ends of the stem-loop structure and thus positions the RNase III A/B domains of Drosha 10-12 nt proximal of the start of the stem-loop structure, which leads to release of the $\sim 55-70 \mathrm{nt}$ pre-miRNA.
The pre-miRNA is then exported from the nucleus by Exp5 (Yi et al., 2003; Bohnsack et al., 2004; Lund et al., 2004). In the crystal structure of the export complex, Exp5 forms a U-like structure that orients the pre-miRNA substrate such that the loop faces the open end of the ' $U$ ' and that the receptor embraces the terminal $16 \mathrm{bp}$ of the pre-miRNA stem (Okada et al., 2009). Molecular dynamics simulations have shown the opening of free Exp5 to allow the binding of RanGTP, which in turn stimulates recruitment of the pre-miRNA by inducing conformational changes and by presenting basic patches for RNA interaction (Wang et al., 2011). Export complex formation is further stimulated by transition to the A-form of the pre-miRNA (Wang et al., 2011), which allows Exp5 to recognise the pre-miRNA stem and $3^{\prime}$ overhang generated by Drosha (Okada et al., 2009). The 3' overhang is typically $2 \mathrm{nt}$ or longer and recognition requires the base flipping of the non-base paired nt (Wang et al., 2011). The mode of recognition of the stem and the $3^{\prime}$ overhang characteristic of RNase III-family products strongly unfavours binding of substrates with shorter stem structures or carrying $5^{\prime}$ overhangs (Zeng and Cullen, 2004). Exp5 largely contacts the RNA backbone and mostly via long-range interactions (shape and charge complementarity), allowing sequenceindependent recruitment and more structural flexibility for the dsRNA cargo (Okada et al., 2009; Güttler and Görlich, 2011; Wang et al., 2011). 
It remains unclear whether the pre-miRNA is directly transferred from the Microprocessor complex to Exp5 for export and also whether there is a channeling mechanism to the RNase III enzyme Dicer after export. Besides its function as an exporter for pre-miRNAs, Exp5 also has the function to stabilise pre-miRNAs as they lack a $5^{\prime}$ cap and a polyA tail at their $3^{\prime}$ end. This is accomplished by binding of the receptor to the termini of its substrate, which would also allow protection without immediate export.

After export complex disassembly in the cytoplasm, the pre-miRNA undergoes a second cleavage step in which the Dicer catalyses the removal of the terminal loop, resulting in a $\sim 22$ nt miRNA duplex with typically 2 nt $3^{\prime}$ overhangs on each side of the dsRNA (reviewed in Miyoshi et al., 2010). The PAZ domain of Dicer recognises the 2 nt $3^{\prime}$ overhang of the pre-miRNA that was generated by Drosha and then acts as a 'molecular ruler' to determine the cleavage sites of its two RNase III domains and to generate miRNAs of defined length. After Dicer cleavage, the microRNA-induced silencing complex (miRISC) loading complex selects the mature miRNA (guide strand) depending on the thermodynamic stability of the base pairing at the two ends of the miRNA duplex, that is, the strand with the lower stability at its $5^{\prime}$ end is selected as guide strand, while the passenger strand is degraded. By partial base pairing, the miRNA in the miRISC then recognises target mRNAs for silencing (see Miyoshi et al., 2010, and references therein).

Besides tRNAs and pre-miRNAs, human Exp5 has been shown to directly interact with the Dicer mRNA (Bennasser et al., 2011). This has been suggested to represent a checkpoint for the miRNA biogenesis pathway, in which export of Dicer mRNA and thus production of Dicer is cross-regulated with export of Dicer substrates.

Various RNA-binding proteins with diverse cellular functions can also be exported by Exp5 in an indirect, piggyback-like fashion. Exp5 was initially shown to export the translation elongation factor eEF1A via aminoacylated tRNA (Bohnsack et al., 2002; Calado et al., 2002) and the interleukin enhancer binding factor 3 (ILF3) (nuclear factor 90) in dependence of its dsRNA-binding domain (dsRBD) (Brownawell and Macara, 2002). In addition, dsRBDs of PKR (dsRBD1), Spnr (dsRBD2) and Staufen (dsRBD3) were shown to be recruited to Exp5 export complexes (Brownawell and Macara, 2002), probably via Exp5-bound dsRNA. In the case of the neuronal Staufen protein family that is involved in mRNA localisation, only Stau2 was later found to be exported by Exp5 in an RNA-dependent manner (Macchi et al., 2004). For ILF3, export was shown to be mediated by Exp5 via RNAs containing minihelix motifs (Gwizdek et al., 2004). Similarly, Exp5 is required for nucleocytoplasmic shuttling of the RNA-binding protein JAZ, which was suggested to be co-exported by Exp5 together with ILF3 (Chen et al., 2004). Additional export substrates of Exp5 include the RNA editing enzyme ADAR1 (Fritz et al., 2009) and the Fas-associated death domain protein (Screaton et al., 2003).

Wild et al. (2010) have performed a screening (RNA interference) for human factors involved in the biogenesis of ribosomal subunits. Interestingly, they found that Exp5 is involved in nuclear export of the pre-60S subunit in human and Xenopus and that the receptor associates with pre-60S co-factors in HeLa cells. It remains to be shown how Exp5 is recruited to pre-60S particles and whether it directly interacts with (pre-)rRNA.

The large majority of Exp5 substrates have been described as export substrates. So far, only one import substrate has been found for an Exp5 homologue, which is the replication protein A that is imported by Msn5 in yeast (Yoshida and Blobel, 2001). Msn5 can thus perform bidirectional transport, which has also been described for Importin 13 and Exportin 4 (reviewed in Güttler and Görlich, 2011).

Emerging evidence has recently linked Exp5 with several diseases, especially various kinds of cancer. While a growing number of reports show that deregulation of miRNA levels often coincides with cellular transformation (see, e.g., Lu et al., 2005), mutations in Exp5 and other proteins involved in the miRNA biogenesis pathway have been found in various cancers with microsatellite instability (Kim et al., 2010; Melo et al., 2010; Leaderer et al., 2011). Melo et al. (2010) have performed a detailed analysis of an Exp5 frame shift mutation that occurs in colorectal cancer using HCT-15 and DLD-1 cell lines. This mutation (a single adenosine insertion at position 3546 of the coding sequence) leads to amino acid changes and truncation of the Exp5 C-terminus. Nuclear accumulation of pre-miRNAs was observed in these cells, which can be rescued by overexpression of wild-type Exp5. Importantly, as Exp5 is also involved in ribosome biogenesis (Wild et al., 2010), the regulation of which is closely linked to cell proliferation and often affected in cancers. This could also contribute to possible cancerogenous effects of the mutation.

Besides cancer, Exp5 levels have been found to be affected in Huntington's disease models (Lee et al., 2011), and several examples of viral 'hijacking' of the receptor have been described. For example, Cazalla et al. (2011) have shown that pre-miRNAs originated from the Herpesvirus saimiri genome after infection of human cells, and that these miRNAs are processed in a Drosha-independent but Exp5- and Dicerdependent manner before integration into the RISC. As another example, the adenoviral VA1 RNA can be exported in an Exp5-dependent manner via a minihelix motif (Gwizdek et al., 2003, 2004). It can be expected that multiple other viruses either manipulate Exp5 activity or make use of this versatile pathway to export large quantities of RNAs for packaging in the cytoplasm.

Taken together, Exp-t has a clearly defined role in the biogenesis of tRNAs, and Exp5 acts in multiple additional transport pathways besides tRNA export. The tRNA biogenesis pathway is best understood in $S$. cerevisiae, but much less is known in other eukaryotes. This particularly applies to the pathway in Drosophila melanogaster, where no Exp-t homologue could be identified so far. Interestingly, D. melanogaster Exp5 was found to have a higher affinity for tRNAs than other Exp5 orthologues (Shibata et al., 2006), suggesting that the receptor has taken over the functions of Exp-t in flies. It is also unclear so far, how Msn5 interacts with tRNAs and whether recruitment of Tef1/2 facilitates export complex 
formation. It will therefore be very interesting to see the molecular structures and contacts in tRNA export complexes not only of human Exp5 but also for some of its orthologues.

It will be exciting to understand how Exp5 can form export complexes with such a large variety of RNA species. It is expected that only a small fraction of the Exp5 substrates are known to date. This not only refers to the recently identified miRNAs that are derived from a large variety of cellular RNAs, such as small nucleolar RNAs, tRNAs and mirtrons (see Miyoshi et al., 2010), but also to many other RNAs as indicated by protein substrates that are co-exported via so far unknown RNAs in different cell types.

As concerns the crosstalk between the different cellular pathways, the concept that individual proteins are often involved in several distinct pathways has widely emerged in recent years. Besides Exp5 itself, several such examples are known for factors acting in the biogenesis of tRNAs (e.g., Utp8 and Utp9, also involved in yeast ribosome biogenesis) and miRNAs (Dicer involvement in ribosomal and spliceosomal RNA maturation in Candida albicans). More such cases are bound to be identified and will allow more levels of cross- or co-regulation of different pathways.

Not much is known so far about the regulation of Exp5 itself. Experimental evidence is accumulating that Exp5 levels are limiting in many cell types and that overexpression of individual substrates can result in a competitive reduction in export of other RNAs (see, e.g., Yi et al., 2005; Bennasser et al., 2011). Exp5 levels have been reported to be influenced by hormones and deregulated in multiple types of cancer (see, e.g., Lu et al., 2005). Regulation of Exp5 activity at the level of individual substrates and pathways could further increase the flexibility of the system. This could be achieved by regulation of the capability of the cargo to bind to the receptor, for example, by masking its Exp5 binding site, or by modulation of the specificity of Exp5 itself. We are curious to see how such mechanisms specifically modulate the activity of this versatile nuclear export receptor.

\section{Acknowledgements}

We thank Carmen Döbele and Thomas Güttler for critical comments on the manuscript and the Deutsche Forschungsgemeinschaft for funding (BO3442/1-1). We also apologise to all colleagues whose work could not be cited due to strict limitations in space and number of references.

\section{References}

Bennasser, Y., Chable-Bessia, C., Triboulet, R., Gibbings, D., Gwizdek, C., Dargemont, C., Kremer, E.J., Voinnet, O., and Benkirane, M. (2011). Competition for XPO5 binding between Dicer mRNA, pre-miRNA and viral RNA regulates human Dicer levels. Nat. Struct. Mol. Biol. 18, 323-327.

Bohnsack, M.T., Regener, K., Schwappach, B., Saffrich, R., Paraskeva, E., Hartmann, E., and Görlich, D. (2002). Exp5 exports eEF1A via tRNA from nuclei and synergizes with other transport pathways to confine translation to the cytoplasm. EMBO J. 21, 6205-6215.
Bohnsack, M.T., Czaplinski, K., and Görlich, D. (2004). Exportin 5 is a RanGTP-dependent dsRNA-binding protein that mediates nuclear export of pre-miRNAs. RNA 10, 185-191.

Brownawell, A.M. and Macara, I.G. (2002). Exportin-5, a novel karyopherin, mediates nuclear export of double-stranded RNA binding proteins. J. Cell Biol. 156, 53-64.

Calado, A., Treichel, N., Müller, E.C., Otto, A., and Kutay, U. (2002). Exportin-5-mediated nuclear export of eukaryotic elongation factor 1A and tRNA. EMBO J. 21, 6216-6224.

Cazalla, D., Xie, M., and Steitz, J.A. (2011). A primate herpesvirus uses the integrator complex to generate viral microRNAs. Mol. Cell 43, 982-992.

Chen, T., Brownawell, A.M., and Macara, I.G. (2004). Nucleocytoplasmic shuttling of JAZ, a new cargo protein for exportin-5. Mol. Cell. Biol. 24, 6608-6619.

Cook, A.G., Fukuhara, N., Jinek, M., and Conti, E. (2009). Structures of the tRNA export factor in the nuclear and cytosolic states. Nature 461, 60-65.

Fritz, J., Strehblow, A., Taschner, A., Schopoff, S., Pasierbek, P., and Jantsch, M.F. (2009). RNA-regulated interaction of transportin-1 and exportin-5 with the double-stranded RNA-binding domain regulates nucleocytoplasmic shuttling of ADAR1. Mol. Cell. Biol. 29, 1487-1497.

Güttler, T. and Görlich, D. (2011). Ran-dependent nuclear export mediators: a structural perspective. EMBO J. 30, 3457-3474.

Gwizdek, C., Ossareh-Nazari, B., Brownawell, A.M., Doglio, A., Bertrand, E., Macara, I.G., and Dargemont, C. (2003). Exportin-5 mediates nuclear export of minihelix-containing RNAs. J. Biol. Chem. 278, 5505-5508.

Gwizdek, C., Ossareh-Nazari, B., Brownawell, A.M., Evers, S., Macara, I.G., and Dargemont, C. (2004). Minihelix-containing RNAs mediate exportin-5-dependent nuclear export of the double-stranded RNA-binding protein ILF3. J. Biol. Chem. 279, 884-891.

Hopper, A.K., Pai, D.A., and Engelke, D.R. (2010). Cellular dynamics of tRNAs and their genes. FEBS Lett. 584, 310-317.

Kim, M.S., Oh, J.E., Kim, Y.R., Park, S.W., Kang, M.R., Kim, S.S., Ahn, C.H., Yoo, N.J., and Lee, S.H. (2010). Somatic mutations and losses of expression of microRNA regulation-related genes AGO2 and TNRC6A in gastric and colorectal cancers. J. Pathol. $221,139-146$.

Köhler, A. and Hurt, E. (2007). Exporting RNA from the nucleus to the cytoplasm. Nat. Rev. Mol. Cell Biol. 8, 761-773.

Leaderer, D., Hoffman, A.E., Zheng, T., Fu, A., Weidhaas, J., Paranjape, T., and Zhu, Y. (2011). Genetic and epigenetic association studies suggest a role of microRNA biogenesis gene exportin-5 (XPO5) in breast tumorigenesis. Int. J. Mol. Epidemiol. Genet. 2, 9-18.

Lee, S.T., Chu, K., Im, W.S., Yoon, H.J., Im, J.Y., Park, J.E., Park, K.H., Jung, K.H., Lee, S.K., Kim, M., et al. (2011). Altered microRNA regulation in Huntington's disease models. Exp. Neurol. 227, 172-179.

Lu, J., Getz, G., Miska, E.A., Alvarez-Saavedra, E., Lamb, J., Peck, D., Sweet-Cordero, A., Ebert, B.L., Mak, R.H., Ferrando, A.A., et al. (2005). MicroRNA expression profiles classify human cancers. Nature 435, 834-838.

Lund, E., Güttinger, S., Calado, A., Dahlberg, J.E., and Kutay, U. (2004). Nuclear export of microRNA precursors. Science 303, 95-98.

Macchi, P., Brownawell, A.M., Grunewald, B., DesGroseillers, L., Macara, I.G., and Kiebler, M.A. (2004). The brain-specific double-stranded RNA-binding protein Staufen2: nucleolar accumulation and isoform-specific exportin-5-dependent export. J. Biol. Chem. 279, 31440-31444. 
Melo, S.A., Moutinho, C., Ropero, S., Calin, G.A., Rossi, S., Spizzo, R., Fernandez, A.F., Davalos, V., Villanueva, A., Montoya, G., et al. (2010). A genetic defect in exportin-5 traps precursor microRNAs in the nucleus of cancer cells. Cancer Cell 18, 303-315.

Miyoshi, K., Miyoshi, T., and Siomi, H. (2010). Many ways to generate microRNA-like small RNAs: non-canonical pathways for microRNA production. Mol. Genet. Genomics 284, 95-103.

Okada, C., Yamashita, E., Lee, S.J., Shibata, S., Katahira, J., Nakagawa, A., Yoneda, Y., and Tsukihara, T. (2009). A high-resolution structure of the pre-microRNA nuclear export machinery. Science 326, 1275-1279.

Screaton, R.A., Kiessling, S., Sansom, O.J., Millar, C.B., Maddison, K., Bird, A., Clarke, A.R., and Frisch, S.M. (2003). Fas-associated death domain protein interacts with methyl-CpG binding domain protein 4: a potential link between genome surveillance and apoptosis. Proc. Natl. Acad. Sci. USA 100, 5211-5216.

Shibata, S., Sasaki, M., Miki, T., Shimamoto, A., Furuichi, Y., Katahira, J., and Yoneda, Y. (2006). Exportin-5 orthologues are functionally divergent among species. Nucleic Acids Res. 34, $4711-4721$.
Wang, X., Xu, X., Ma, Z., Huo, Y., Xiao, Z., Li, Y., and Wang, Y. (2011). Dynamic mechanisms for pre-miRNA binding and export by Exportin-5. RNA 17, 1511-1528.

Wild, T., Horvath, P., Wyler, E., Widmann, B., Badertscher, L., Zemp, I., Kozak, K., Csucs, G., Lund, E., and Kutay, U. (2010). A protein inventory of human ribosome biogenesis reveals an essential function of exportin 5 in 60S subunit export. PLoS Biol. 8, e1000522.

Yi, R., Qin, Y., Macara, I.G., and Cullen, B.R. (2003). Exportin-5 mediates the nuclear export of pre-microRNAs and short hairpin RNAs. Genes Dev. 17, 3011-3016.

Yi, R., Doehle, B.P., Qin, Y., Macara, I.G., and Cullen, B.R. (2005). Overexpression of exportin 5 enhances RNA interference mediated by short hairpin RNAs and microRNAs. RNA 11, 220-226.

Yoshida, K. and Blobel, G. (2001). The karyopherin Kap142p/ Msn5p mediates nuclear import and nuclear export of different cargo proteins. J. Cell Biol. 152, 729-740.

Zeng, Y. and Cullen, B.R. (2004). Structural requirements for premicroRNA binding and nuclear export by Exportin 5. Nucleic Acids Res. 32, 4776-4785.

Received March 6, 2012; accepted April 4, 2012 